\title{
Self-induced magnetic field effects caused by edge currents in parallel array of Josephson junctions
}

Kaplunenko, V. K.; Goldobin, E. B.; Khabipov, M. I.; Larsen, Britt Hvolbæk; Mygind, Jesper; Pedersen, Niels Falsig

Published in:

Journal of Applied Physics

Link to article, DOI:

$10.1063 / 1.354178$

Publication date:

1993

Document Version

Publisher's PDF, also known as Version of record

Link back to DTU Orbit

Citation (APA):

Kaplunenko, V. K., Goldobin, E. B., Khabipov, M. I., Larsen, B. H., Mygind, J., \& Pedersen, N. F. (1993). Selfinduced magnetic field effects caused by edge currents in parallel array of Josephson junctions. Journal of Applied Physics, 74(9), 5854-5858. https://doi.org/10.1063/1.354178

\section{General rights}

Copyright and moral rights for the publications made accessible in the public portal are retained by the authors and/or other copyright owners and it is a condition of accessing publications that users recognise and abide by the legal requirements associated with these rights.

- Users may download and print one copy of any publication from the public portal for the purpose of private study or research.

- You may not further distribute the material or use it for any profit-making activity or commercial gain

- You may freely distribute the URL identifying the publication in the public portal 


\title{
Self-induced magnetic field effects caused by edge currents in parallel array of Josephson junctions
}

\author{
V. K. Kaplunenko, E. B. Goldobin, and M. I. Khabipov \\ Institute of Radio Engineering and Electronics, RAS, Moscow, Russia \\ Britt H. Larsen, J. Mygind, and N. F. Pedersen \\ Physics Department, Technical University of Denmark, Lyngby, Denmark
}

(Received 23 March 1993; accepted for publication 13 July 1993)

\begin{abstract}
A transmission line consisting of a parallel connection of shunted Josephson junctions is one of the base elements in the new Rapid Single Flux Quantum (RSFQ) logic. When an additional bias current is applied to the edge junction of the transmission line it generates and injects series of flux quanta on the line. This kind of pulse generators have important applications for testing RSFQ devices at high frequencies. In such experiments unwanted steps always show up in the $I-V$ curve of the generator junction. This problem has been experimentally and numerically investigated for parallel arrays of identical junctions. Steps with extremely low differential resistance are found to be due to the self-induced magnetic field produced by the edge current. The underlying mechanism is that the non-uniform field divides the array into domains consisting of several (unit) cells each containing the same number of flux quanta. The influence of an applied homogeneous external magnetic field and a uniformly distributed bias current is also considered.
\end{abstract}

\section{INTRODUCTION}

A transmission line consisting of a parallel connection of shunted Josephson junctions is one of the base elements in the new Rapid Single Flux Quantum (RSFQ) logic, where much progress has been reported recently. ${ }^{1}$ The same type of circuits has also been used for many other applications in superconducting electronics, such as for example amplifiers and flux-flow transistors. ${ }^{2}$ The propagation of single flux quanta (SFQ) through a resistively shunted Josephson junction is the fundamental process in RSFQ circuits. One of the base elements of RSFQ logic is the transmission line consisting of a parallel connection of shunted Josephson junctions. This line may be employed to transfer the SFQ pulses between active elements, to amplify the magnetic field energy connected with the flux quantum, ${ }^{1}$ and to provide a time delay of fluxon propagation..$^{3-6}$ The high frequency properties of RSFQ devices can be tested by rather simple dc measurements ${ }^{4,7}$ relying on the Josephson relation, $f=(2 e / h) V_{\mathrm{dc}}$, which relates the frequency, $f$, of the internal Josephson oscillation to the average voltage, $V_{\mathrm{dc}}$, across the junction.

The parallel junction array may also be used as a SFQ generator. When an additional bias current is applied to the edge junction it generates flux quanta, which propagate down the transmission line. Unfortunately a number of characteristic steps appear in the $I-V$ curve of the fluxon generator junction. These steps have practically zero differential resistance and render the analysis of RSFQ circuits more difficult. In the discussion of our experiments we show that neither internal junction (geometric) resonances nor cavity resonances (in the loop formed by the junction capacitance and the inductances of the superconducting strips connecting the junctions) are responsible for these steps. The extremely low differential resistance of the steps cannot be explained by the usual two junction inter- ferometer model. The main aim of this paper is to determine the nature of these steps. The same effect may have been observed recently in an experiment by van der Zant et $a l^{8}{ }^{8}$ They investigated the dynamics of fiuxons in underdamped junction arrays. Close to the transition temperature (where the damping becomes large as in our experiment) they found similar steps in the $I-V$ curve. They tentatively related the observed steps to Fiske resonances; however, based on our observations, non-uniform current feeding as discussed below, might also be responsible for the steps.

\section{EQUIVALENT CIRCUIT}

Figure 1 shows the equivalent diagram of the circuit under investigation. The one-dimensional array consists of twenty parallel connected Josephson junctions $\left(J_{1}-J_{20}\right)$ each of which are externally resistively shunted to provide a McCumber parameter, $\beta_{c} \approx 1$. The unit cell is a twojunction interferometer sharing its junctions with the neighboring cells. There are three bias currents supplied to the array. $I_{b}$ is a uniformly distributed current applied to the individual junctions through the resistances $R_{1}-R_{20}$. These resistances are ten times larger than the junction shunt resistances. $I_{e 1}$ is fed directly to the edge junction $J_{1} . I_{e 2}$ is fed directly to $J_{20}$ and is used only when applying a magnetic field, which is controlled by adding a dc current, $I_{h}$, to $I_{e 1}$ and having $I_{e 2}=I_{h}$ at $J_{20}$. The array voltage, $V$, is measured across one of the edge junctions.

\section{FABRICATION TECHNIQUE}

Standard trilayer $\mathrm{Nb}-\mathrm{AlO}_{x}-\mathrm{Nb}$ technique was employed to fabricate the 20-junction arrays with a critical current density of about $1 \mathrm{kA} / \mathrm{cm}^{2}$. The junctions were identical with the following characteristics: Critical current 


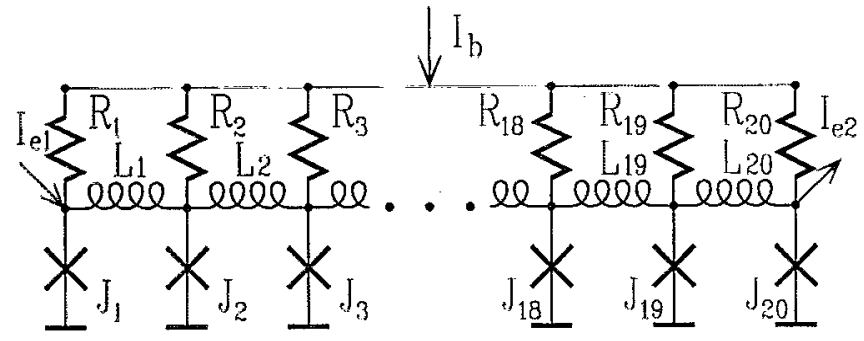

FIG. 1. Equivalent circuit of one-dimensional array of over-damped Josephson tunnel junctions (crosses). The resistances $R_{1}-R_{20}$ provide a uniform bias current. A magnetic field may be introduced by adding a current, $I_{h}$, to one of the edge currents, say $I_{e 1}$ and subtracting it from the other, $I_{e 2}$. The array voltage, $V$, is measured across one of the edge junctions.

$I_{c}=265 \mu \mathrm{A}$, junction resistance (including the external shunt resistance) $R=1.75 \Omega$. The junction capacitance is estimated to be $C=0.8 \mathrm{pF},{ }^{9}$ giving a McCumber parameter $\beta_{c}=2$. We have estimated the capacitance connected with the overlap geometry to be negligible. This is confirmed by measurements using different geometries. The inductances, $L_{1}-L_{20}$, as measured with a two-junction interferometer located on the substrate close to the array had the selfinductance $L=9.4 p \mathrm{H}$. The junctions were circular with a diameter of $5 \mu \mathrm{m}$.

\section{EXPERIMENTAL RESULTS}

The measured $V\left(I_{b}\right)$ curve with fixed $I_{e 1}$ appears as a smooth curve that accurately fits to the predictions of the Resistively Shunted Junction (RSJ) model for a single junction, $V=R_{t}\left(I_{b}^{2}-I_{c}^{2}\right)^{1 / 2}$; here $R_{t}$ is the total shunt resistance of the junctions in the array and $I_{c}$ is a function of $I_{e 1}$. An external magnetic field introduced by $I_{h}$ (with fixed edge current $I_{e 1}$ as described above) does not change the shape of the $I-V$ characteristic. It means that the bias current $I_{b}$ is uniform and does not influence the magnetic field distribution in the array. On the contrary, an applied edge current $I_{e 1}$ significantly changes this distribution. The

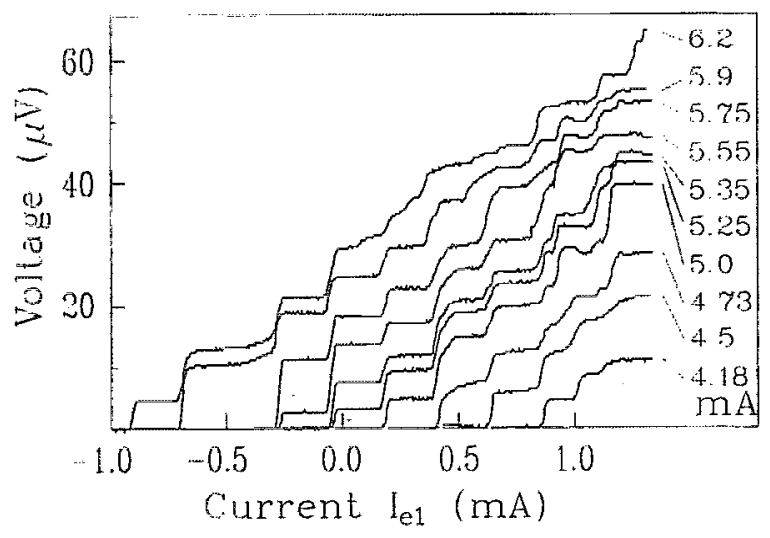

FIG. 2. Array voltage, $V$, vs edge current, $I_{e 1}$, measured for different values of the common bias current, $I_{b}$, for zero external magnetic field $\left(I_{h}=0, I_{e 2}=0\right)$.

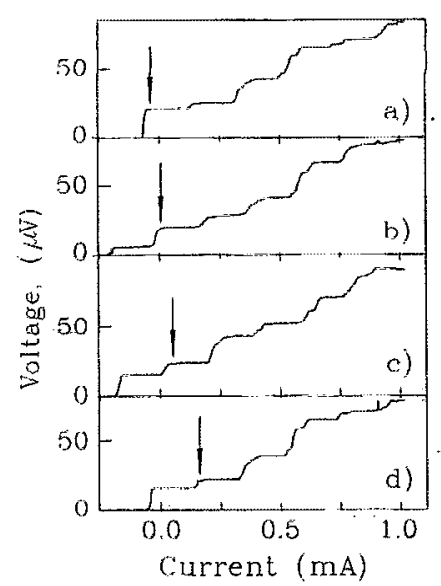

FIG. 3. Experimental $V\left(I_{e 1}\right)$ curves at different values of the magnetic field and fixed common bias current, $I_{b}=5.2 \mathrm{~mA}$; (a) $I_{h}=0.4 \mathrm{~mA}$, (b) $I_{h}=0.31 \mathrm{~mA}$, (c) $I_{h}=0.22 \mathrm{~mA}$, and (d) $I_{h}=0.07 \mathrm{~mA}$. The arrow indicates the shift of the same step.

experimental $V\left(I_{e 1}\right)$ curves obtained with zero magnetic field $\left(I_{h}=0\right)$ for ten different fixed values of $I_{b}$ are presented in Fig. 2.

The bias current, $I_{b}$, defines the velocity of fluxons moving through the array and influences the voltage positions of the (nearly) horizontal steps. From the experimental curves we have found that the current width, $\Delta I_{e 1}$, of the steps is given by $\Delta I_{e 1}=\Phi_{0} / L$, where $\Phi_{0}=h /(2 e)$ is the magnetic flux quantum and $L$ is the inductance connecting the junctions. The numerical simulations confirmed this observation. Accordingly, a new step appears when an additional fluxon penetrates into the array. This effect is similar to the one observed for a nonsymmetrically biased two-junction interferometer, where the bias current induces a magnetic field in the interferometer loop, resulting in the well known modulation of the $I-V$ curve. The extremely small differential resistance of the steps in the junction array may be interpreted as being due to some kind of nonlinear resonant interaction. It is definitely not a geometrical resonance since the step voltage varies with the common bias current $I_{b}$ (as given

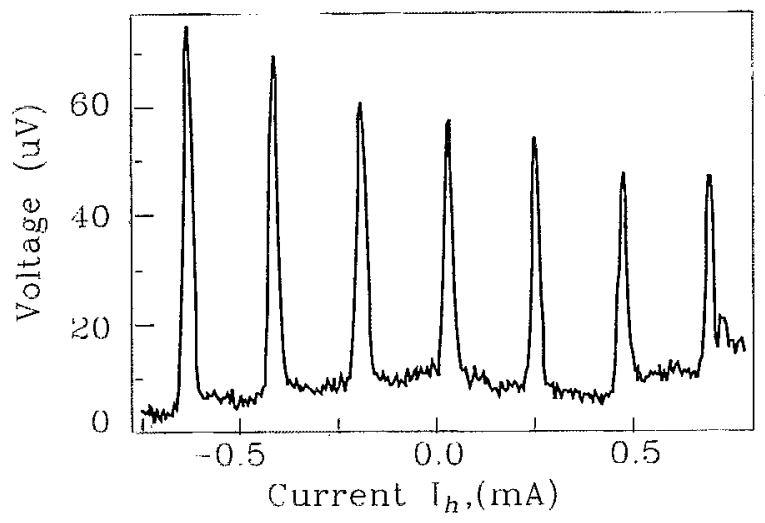

FIG. 4. Array voltage as function of magnetic field for fixed $I_{b}=5.2 \mathrm{~mA}$ and $I_{e 1}=-0.22 \mathrm{~mA}$. 


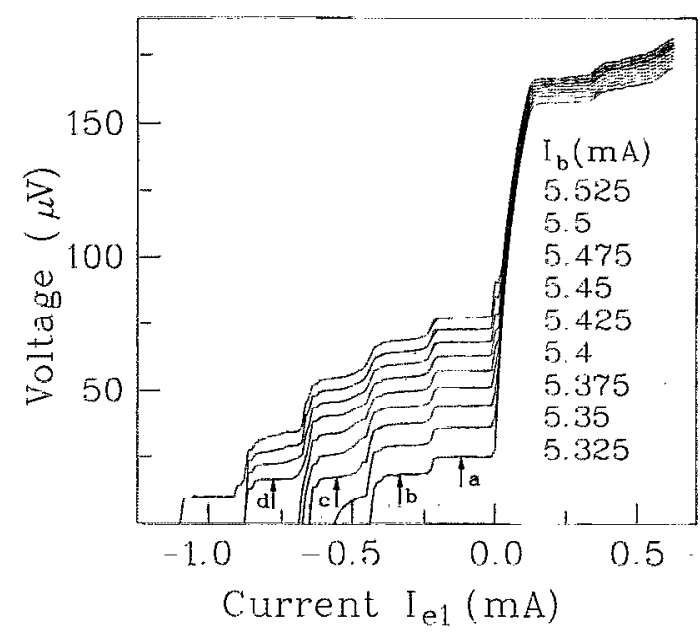

FIG. 5. Computer simulation using the experimental parameter values of the circuit elements. Calculated $V\left(I_{e 1}\right)$ curve for different values of the tolal uniform bias current, $I_{b}$. The step voltages are strictly proportional to $\left(I_{b}^{2}-I_{c}^{2}\right)^{1 / 2}$.

above), and also because the $V\left(I_{b}\right)$ characteristic is smooth. Furthermore the damping of the circuit is much too large for the observation of geometrical resonances. It seems as if the array is screening the applied magnetic field and only permits an integer number of fluxons to enter the array.

Figures 3(a)-3(d) show a family of $V\left(I_{e 1}\right)$ curves for different values of the uniformly applied magnetic field (fixed $I_{h}$ ). It is obvious that decreasing the magnetic field shifts the steps towards larger $I_{e 1}$ values. Actually, experiments show that a new step appears whenever the magnetic field is incremented by $\Delta I_{h}=\Phi_{0} / L$, corresponding to one more fluxon threading the array [see Figs. 3(a) $-3(b)$ ]. In the range $\Delta I_{h}$ the magnetic field is unable to change the number of fluxons in the array.

Figure 4 shows the array voltage as function of magnetic field at the fixed edge current, $I_{e 1}$, where the steps appeared in Fig. 3(c). One can see that each new step reduces the critical current and results in an increase of the voltage. Each time after the step has moved on to the right in Fig. 3 the critical current becomes large again.

\section{COMPUTER SIMULATION}

The 20-junction array has been simulated within the framework of the simple RSJ model as justified by the small shunt resistance. The junction parameters used in the simulations were close to the experimental values and are: Critical current $265 \mu \mathrm{A}$, resistance $1 \Omega$, capacitance 0.8 $\mathrm{pF}$, and inductance $9.4 \mathrm{pH}$. The sample design was made in such a way that we avoided the influence of the microstrip
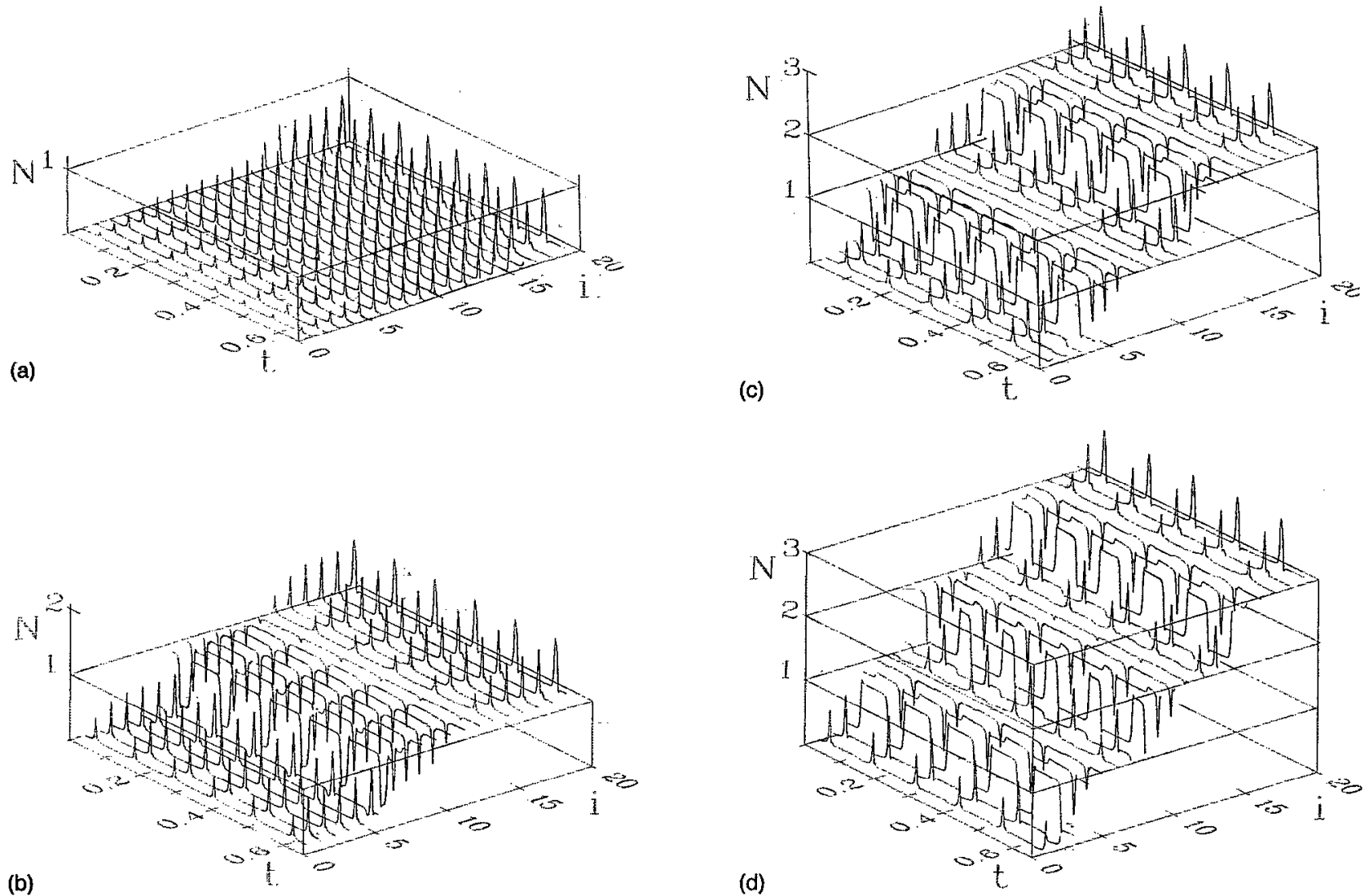

(c)

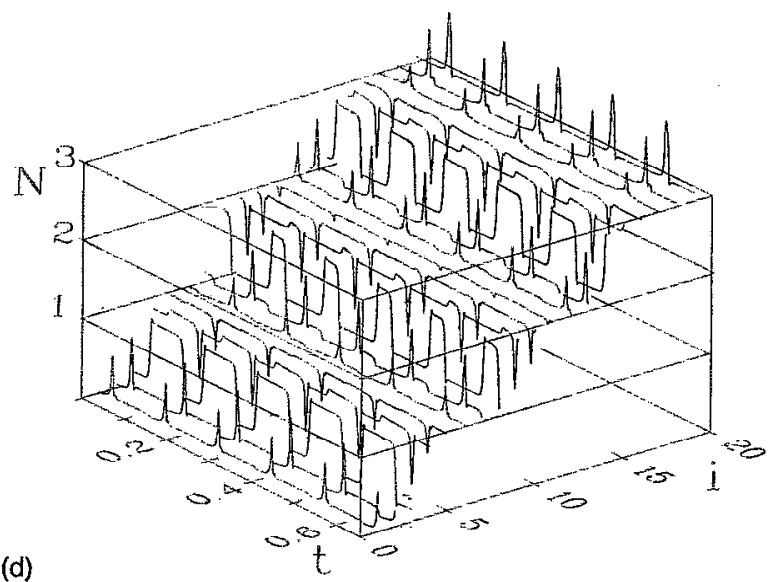

FIG. 6. Number of fluxons $(N)$ located in a unit cell as function of cell number, $i$, and time, $t$. The time unit is 1 ns. The corresponding bias points are marked a-d in Fig. 5. The edge current was introduced to the 20 th junction. 

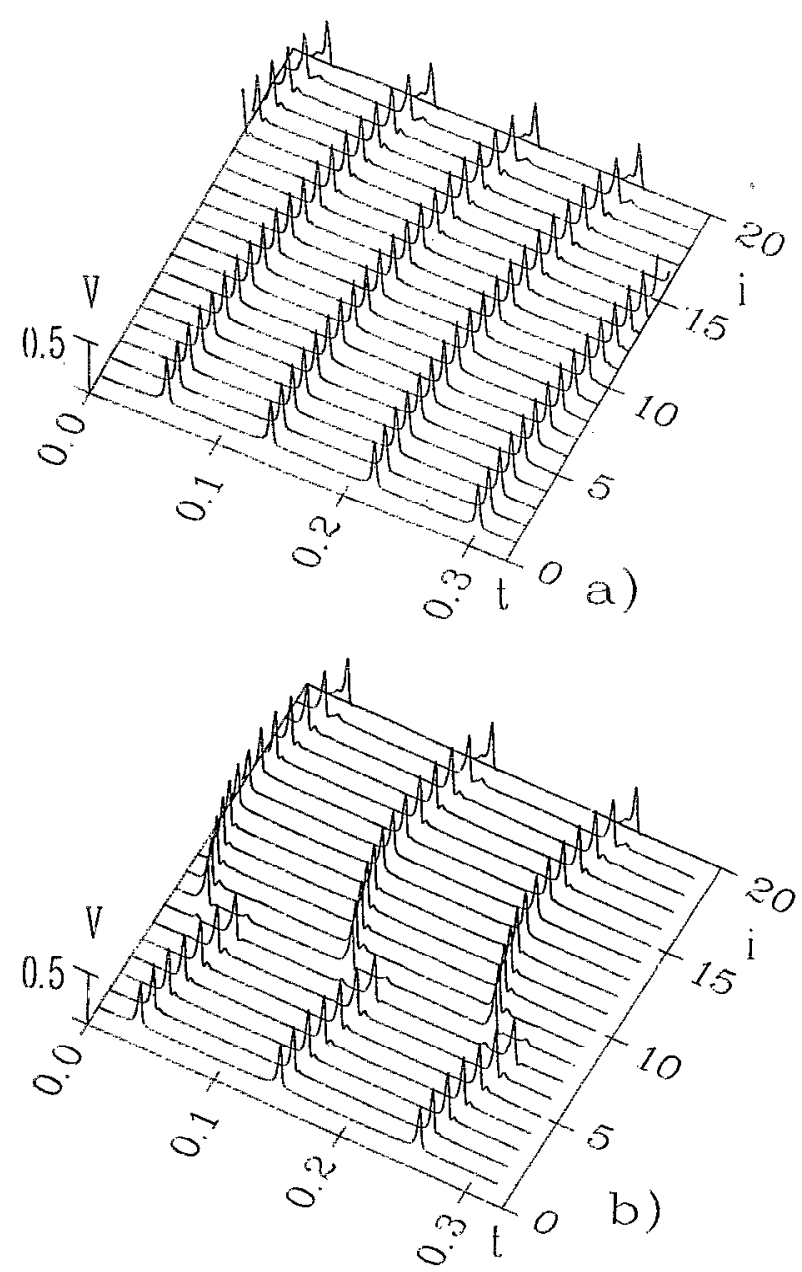
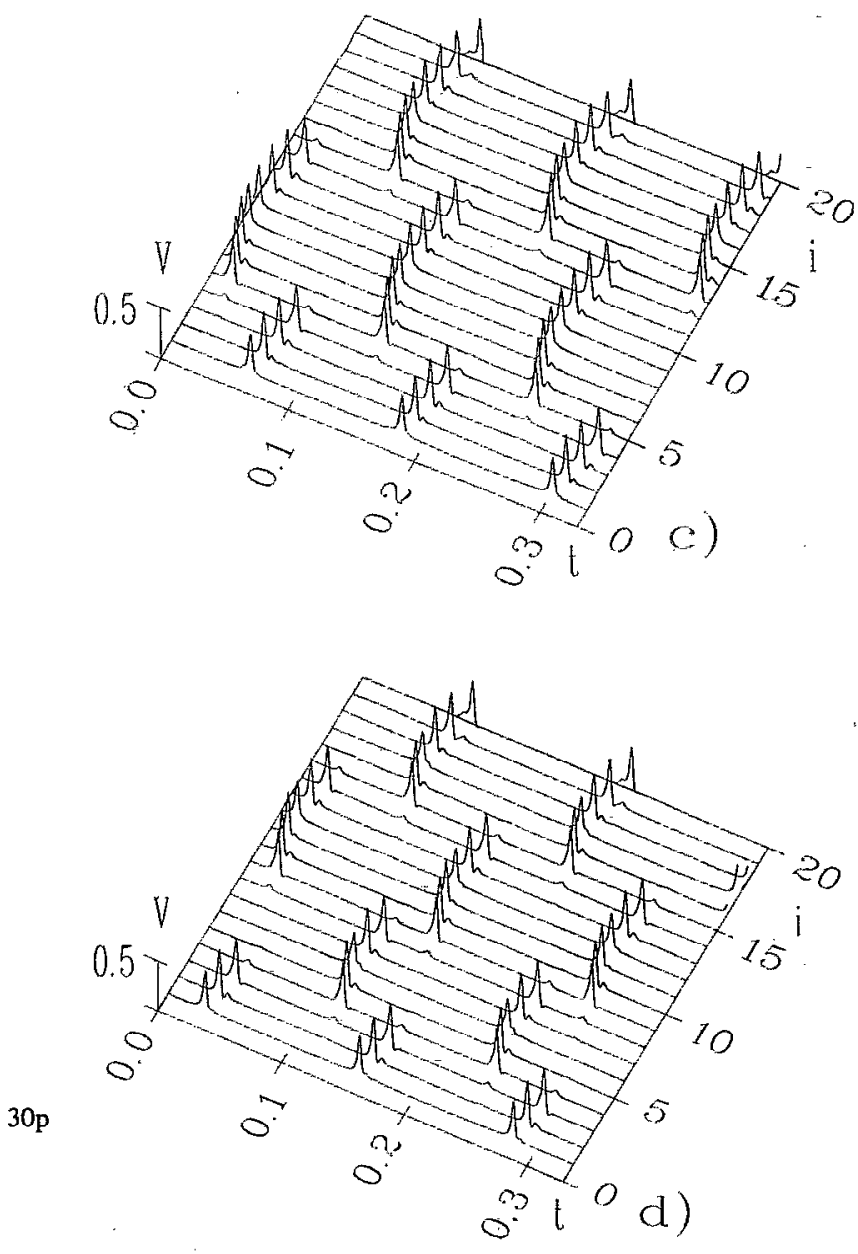

FIG. 7. Voltage (in $\mathrm{mV}$ ) across individual junctions as function of its number, $i$, and time, $t$. The time unit is $1 \mathrm{~ns}$. The edge current is introduced to the 20th junction.

lines used in the circuit. That was done by careful use of filters, damping parameters and geometrical dimensions. If these precautions were not taken resonances due to such effects were easily observed in experiment. Hence in our simulations we do not include such effects.

Figure 5 shows the calculated $V\left(I_{e 1}\right)$ curves at different values of the common bias current, $I_{b}$. There is good general agreement between the simulations and the experimental curves in Fig. 2. We note that the size of the steps and their voltage position agree between simulation and experiment. The same simulation made zero capacitance gave the same $I-V$ curve, indicating that the steps are not caused by any LC resonance. We speculate that the discrepancy for $I_{e 1}>0$ may be caused by an additional parasitic magnetic field in the experiment, or possibly accumulation of numerical errors (double precision) in the simulations, which were rather time consuming especially between steps.

In order to understand the processes causing the step structure, two kinds of curves with bias points indicated a-d in Fig. 5 were calculated. The first is shown in Figs. 6(a) -6 (d) presenting the quantity $N=\left(\phi_{i+1}-\phi_{i}\right) / 2 \pi$ as a function of cell number, $i$, and time, $t$, where $\phi_{i}$ is the quantum-mechanical phase of junction $i$. $N$ thus becomes the number of fluxons in the cell defined by junctions $i$ and $i+1$, i.e., $N=\Phi / \Phi_{0}$. By its definition $N$ may take noninteger values during the transient flux propagation as can be seen in Fig. 6. Figures 7(a)-7(d) show the instantaneous voltage across the individual junctions as function of the same arguments. The voltage pulses observed appcar when a fluxon is transferred from one cell to the next. From Fig. 6 and Fig. 7 we see that the edge current causes a splitting of the array into sections (domains) each having predominantly the same number of fluxons per unit cell.

One can see that the non-uniform magnetic field caused by the edge current penetrates into the array over a distance significantly larger than the Josephson penetration depth observed in long tunnel junctions. This is somewhat unexpected since the ratio of the Josephson inductance to the geometric inductance is only about 0.12 . The first step [see Fig. 6(a) and Fig. 7(a)] seems to be due only to the edge current which is constant and dictates the repetition rate of the fluxon excitation. The step voltage depends on this repetition rate and on the average velocity of the fluxon. This also explains the increase of the step voltage with increasing total bias current depicted in Fig. 2 and Fig. 5.

A more complex picture appears for higher order 
steps. In Figs. 6(b) -6 (d) and Figs. 7(b) -7 (d) one can see that we have two types of domains; the one near the left edge looks like the one discussed above. The domains not reaching the edges are spatially symmetric; the junction in the middle of the domain (in the following called the leading junction) switches first, producing a fluxon and an antifluxon that in turn propagate in opposite directions. That means that the total fluxon number within a symmetrical domain is conserved at all times. This intrinsic feature characterizes all the symmetrical domains in Figs. 6(b)6(d). The simulation shows that the number of domains changes as we go from one step to another, and that a variation of the edge current within the same voltage step only changes the spatial position of the leading junction in the edge domains. It looks as if there is a mutual phaselocking between the leading junctions belonging to different domains. The junctions are connected by a superconductor and must be synchronized, leaving only the spatial location of the leading junction as the free parameter.

\section{CONCLUSION}

We have computer simulated and experimentally investigated the new effect of non-uniform magnetic field penetration into a one-dimensional array of over-damped Josephson junctions. Many of the experimental features have been found in the simulations but obviously a more reliable theory is required in order to analyze complex RSFQ circuits. The theoretical description of the nature of the effect is still open and it is not clear whether the effect is due to a non-uniform current distribution, a mutual phase-locking between domains, or some other mechanisms.

Self-induced magnetic field effects can be also observed in non-uniform arrays and in magnetically extended Josephson structures such as the novel high- $T_{c}$ junctions.
The inhomogeneity of these junctions produces a complicated magnetic field distribution which in turn may result in the peculiarities seen in their $I-V$ characteristics. Investigation of this problem by computer simulations is planned in the near future.

The understanding of the effects described here also seems to offer an explanation of the deficiencies in the high frequency operation ${ }^{6}$ of the Josephson sampler. The presence of domains on the transmission line causes an altered dependence of the time delay on the input voltage.

\section{ACKNOWLEDGMENTS}

The samples were fabricated in the IRE Technology Group headed by professor V. P. Koshelets. We also thank Olga V. Kaplunenko and S. Kovtonuk who participated in the circuit fabrication, and A. V. Ustinov for useful discussions. This work was partially supported by the Danish Research Academy.

${ }^{1}$ K. K. Likharev and V. K. Semenov, IEEE Supercond. 1, 13 (1991).

${ }^{2}$ J. S. Martens, V. M. Hietala, T. A. Plut, D. S. Ginley, G. A. Vawter, C. P. Tigges, M. P. Siegal, J. M. Phillips, and S. Y. Hou, IEEE Trans. Appl. Supercond. 3, 2295 (1993).

${ }^{3}$ V. K. Kaplunenko, M. I. Khabipov, V. P. Koshelets, K. K. Likharev, O. A. Mukhanov, I. L. Serpuchenko, and A. N. Vystavkin, IEEE Trans. Magn. MAG-25, 861 (1989).

${ }^{4}$ V. K. Kaplunenko, L. V. Filipenko, M. I. Khabipov, V. P. Koshelets, K. K. Likharev, S. V. Rylov, V. K. Semenov, and A. N. Vystavkin, IEEE Trans. Magn. MAG-27, 2464 (1991).

${ }^{5}$ V. K. Kaplunenko, M. I. Khabipov, and E. B. Goldobin, Supercond. Sci. Technol. 4, 674 (1991).

${ }^{6}$ E. B. Goldobin, V. K. Kaplunenko, M. I. Khabipov, and L. V. Filipenko, Cryogenics 32, 549 (1992).

${ }^{7}$ V. M. Golomidov, V. K. Kaplunenko, M. I. Khabipov, V. P. Koshelets, and O. V. Kaplunenko, Cryogenics 32, 509 (1992).

${ }^{8}$ H. S. J. van der Zant, E. H. Visser, D. R. Curd, T. P. Orlando, and K. A. Delin, IEEE Trans. Appl. Supercond. 3, 2638 (1993).

${ }^{9}$ V. P. Koshelets, S. A. Kovtonyuk, I. L. Serpuchenko, L. V. Filippenko, and A. V. Shchukin, IEEE Trans. Magn. MAG-27, 3141 (1990). 